\title{
Gamma-ray flares from AGN jets colliding with luminous stars
}

\section{Piotr Banasiński*}

Department of Astrophysics, University of Lodz, Lodz, Poland E-mail: p.banasinski@uni.lodz.pl

\section{Wlodek Bednarek}

Department of Astrophysics, University of Lodz, Lodz, Poland

E-mail: bednar@uni.lodz.pl

\section{Julian Sitarek}

Department of Astrophysics, University of Lodz, Lodz, Poland E-mail: j.sitarekeuni.lodz.pl

\begin{abstract}
We consider a model in which $\gamma$-rays in blazars are produced during encounters of relativistic blobs of plasma with radiation field produced by luminous stars within the jet. The blob, formed in the inner part of the jet, already contains relativistic electrons which comptonize stellar radiation to $\mathrm{GeV}$-TeV $\gamma$-ray energies. These $\gamma$-rays can initiate the Inverse Compton $e^{ \pm}$pair cascade in the radiation field of the star within the jet. Such a scenario might be responsible for the appearance of short time scale $\gamma$-ray flares. The star collision model can explain the appearance of the extreme orphan $\gamma$-ray flare observed in the $\mathrm{GeV}$ and sub-TeV energy range from the flat spectrum radio quasar PKS 1222+21.
\end{abstract}

35th International Cosmic Ray Conference - ICRC2017-

10-20 July, 2017

Bexco, Busan, Korea

\footnotetext{
* Speaker.
} 


\section{Introduction}

The non-thermal radiation in blazars is expected to be produced within a relativistic jet directed towards the observer. Recently, simple scenarios exploiting collisions of compact objects with the jet plasma reached some attention. In fact, many different types of compact objects (e.g. stars, clouds or even globular clusters, fragments of supernova remnants or pulsar wind nebulae) can be immersed within the jet, forming an obstacle for the jet plasma. It has been proposed that particles, accelerated on the shocks appearing as a result of collisions of the jet plasma with stellar winds, can produce high energy $\gamma$-rays (see e.g. [1, 2, 3, 4, 5, 6, 7, 8]). We consider another encounter scenario in which relativistic electrons, already present in the fast moving blob, interact with the dense radiation of a star within the jet. The radiation field of the star is strong enough that $\gamma$-rays produced in the Inverse Compton process can initiate an $e^{ \pm}$pair cascade in the stellar radiation. We developed a dedicated Monte Carlo code tracking such a cascade in the anisotropic radiation field of a star. As an example, we show that the $\gamma$-ray spectrum and the light curve, observed from a distant Flat Spectrum Radio Quasar (FSRQ) PKS 1222+21 during its 2010 orphan $\gamma$-ray flare, might be explained in terms of such a scenario [ [9].

We propose that blobs of relativistic plasma, moving in jets of active galactic nuclei (AGNs), can encounter from time to time the luminous stars which form a quasi-spherical halo around the central super-massive black hole. For example, in the nearby active radio galaxy Cen $\mathrm{A}$, the current star formation rate $\left(\sim 2 \mathrm{M}_{\odot} \mathrm{yr}^{-1}\right)$ should result in the production of $(6-12) \times 10^{7} \mathrm{M}_{\odot}$ of young stars during the duty cycle of the Cen A active nucleus. We can thus expect $\sim 3 \times 10^{5}$ stars with masses above $20 \mathrm{M}_{\odot}$ [5]. A large number of these stars should be immersed in the jet pointing towards the observer. In the case of a simple conical jet, the number of stars is estimated to be of the order of $\sim 83 \gamma_{30}^{-2}$, where we assumed that the jet opening angle is determined by the Lorentz factor of the flow $\theta \sim 1 / \gamma_{b}$ and $\gamma_{b}=30 \times \gamma_{30}$. The Cen A nucleus is also immersed in a quasispherical bulge of late type stars with the number estimated as $\sim 8 \times 10^{8}$ [5]. The total number of luminous red giants can be as large as $\sim 10^{6}$, if only $\sim 10^{-3}$ of these bulge stars are in the red giant phase. Therefore, the interaction of a relativistic blob with the radiation field of such luminous stars within a jet of an active galaxy seems to be quite likely [9].

\section{Gamma-rays from IC pair cascade}

We assume that the blob, a quasi-cylindrical region within the jet containing relativistic electrons, moves along the jet with a fixed Lorentz factor $\gamma_{\mathrm{b}}$. The blob is characterised by its radius $R_{\mathrm{b}}$ and longitudinal extend, $H_{\mathrm{b}}$ (measured in the reference frame of the star). The radius of the blob has to be smaller than the local perpendicular extend of the jet $R_{\perp}$. In the case of a simple conical jet, $R_{\perp} \approx \theta l=10^{16} \gamma_{30}^{-1} l_{-1} \mathrm{~cm}$, where $l=0.1 l_{-1} \mathrm{pc}$ is the distance from the jet base. We assume that the longitudinal extend of relativistic electrons within the blob can be described by a Gaussian distribution with a standard deviation of $H_{\mathrm{b}}$. Such a blob, when passing the stellar radiation, assumed to be point like, should produce a flare on the time scale determined by $H_{\mathrm{b}}$. Note that the emission is produced by successive layers of the blob as they enter in the fixed volume around the star, rather than from the whole blob traversing the jet. Thus, the observed duration of the emission is not going to be shortened by large values of $\gamma_{b}$. 

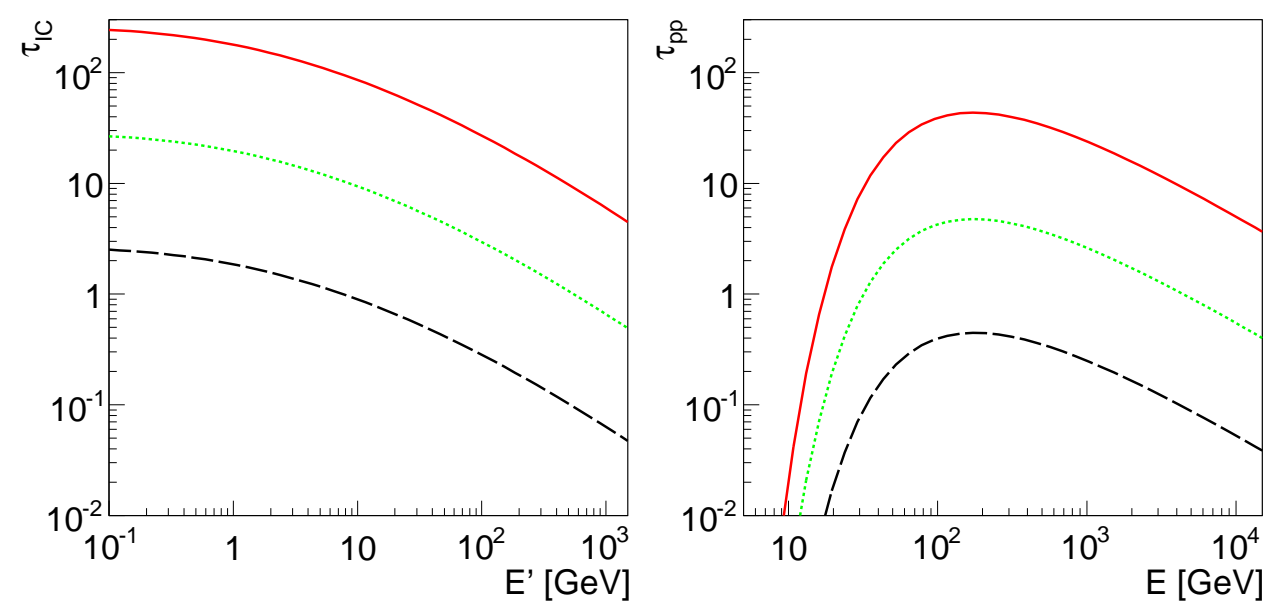

Figure 1: Optical depth for the inverse Compton scattering of the stellar radiation by electrons in the blob (the left panel) and for absorption of produced $\gamma$-rays in this stellar radiation (the right panel) as the function of the energy (measured in the reference frame of the blob with $\gamma_{b}=10$ for the electrons and in the reference frame of the star for the $\gamma$-rays). The impact distance, $d$, is equal to $1.1 R_{\star}$ (red, solid lines), $10 R_{\star}$ (green dotted) and $100 R_{\star}$ (black dashed), where $R_{\star}=10^{12} \mathrm{~cm}$. (reproduced from [9])

Relativistic electrons in the blob can suffer strong energy losses on comptonization of radiation coming from a single star provided that the star is close to the direction of a moving blob. We assume that electrons are already injected into the blob with a power law spectrum. They are isotropic in the blob reference frame and the blob moves relativistically through the jet. The acceleration mechanism of electrons and their maximum energies are not specified in our model. They depend on the acceleration efficiency of electrons and of their dominant energy loss process. We assume that electrons can reach at least sub- $\mathrm{TeV}$ energies which in the case of the acceleration process saturated by the synchrotron energy losses would require the magnetic field within the blob below several Gauss (see e.g. Eq. 3 in [11]). Electrons with sub-TeV energies in the relativistic blob can scatter the stellar radiation already quite deep in the Klein-Nishina (KN) regime. Therefore, $\gamma$-ray photons produced in the IC process can have energies comparable to energies of the primary electrons. These $\gamma$-rays can be again absorbed in this same radiation field, initiating an IC $e^{ \pm}$pair cascade in the radiation of the luminous star.

Let us consider a single electron at a specific distance, $L$, from the perpendicular plane containing the star and with the impact parameter $d$. The direction of the electron is isotropised in the blob by the random magnetic field. In order to determine whether the electron can produce a $\gamma$-ray photon and whether the photon can be absorbed in the stellar radiation, we calculate their IC scattering mean free path and the $\gamma-\gamma \rightarrow e^{ \pm}$mean free path for specific impact distances. In Fig. 2 we show the optical depth for the Inverse Compton (IC) process of electrons on the stellar radiation as the function of their energy measured in the blob's frame of reference. In the calculations, as an example, we assume an $\mathrm{O}$ type star with a temperature $T_{\star}=3 \times 10^{4} \mathrm{~K}$ and a radius of $R_{\star}=10^{12} \mathrm{~cm}$. The optical depth is inversely proportional to $d$. The electrons can efficiently produce $\gamma$-rays up to a distance $d \sim 10^{14} \mathrm{~cm}$. At the highest energies the optical depth is diminished by the $\mathrm{KN}$ effect. The right panel of Fig. 2 shows the optical depth for the absorption of $\gamma$-rays in the $e^{ \pm}$pair production process. For the assumed temperature of the star the peak of the pair production cross section 

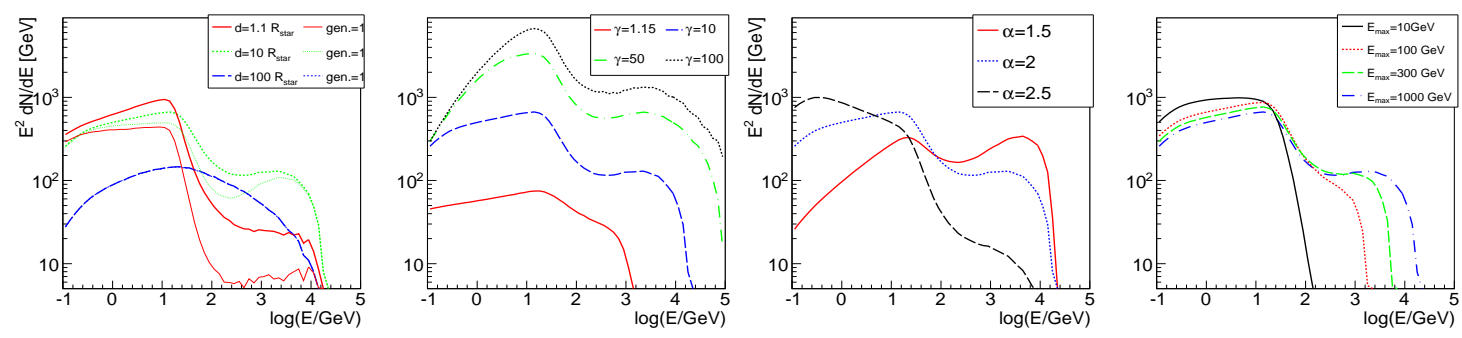

Figure 2: Spectral energy distribution (SED) of the $\gamma$-ray spectrum for a power law differential spectrum of electrons with an spectral index of $\alpha$ between $0.1 \mathrm{GeV}$ and $E_{\max }^{\prime}$ for fixed impact distance of electrons. First figure (from the left): dependence on impact distance $d=1.1 R_{\star}$ (red solid), $10 R_{\star}$ (green dotted), $100 R_{\star}$ (blue dashed), thick lines show the spectra from the full cascade, thin lines show the spectra escaping from the first generation of photons. Second figure: dependence on the Lorentz factor of the blob $\gamma_{b}=1.15$ (red solid), 10 (blue dashed), 50 (green dot-dashed) and 100 (black dotted). Third figure: dependence on the spectral index of electrons $\alpha=1.5$ (red solid), 2 (blue dotted) 2.5 (black dashed). Forth figure: dependence on the maximum energy of the electrons (measured in the blob's frame): $E_{\max }^{\prime}=10 \mathrm{GeV}$ (black solid), $100 \mathrm{GeV}$ (red dotted), $300 \mathrm{GeV}$ (green dashed), $1000 \mathrm{GeV}$ (blue dot-dashed). Unless specified otherwise $\gamma_{b}=10, d=10 R_{\star}, \alpha=2, E_{\max }^{\prime}=1 \mathrm{TeV}$. The spectra are normalised to $1 \mathrm{erg}$ of injected electron energy in the blob's frame of reference. (reproduced from [])

falls around $100 \mathrm{GeV}$. Large values of the optical depth lead to a strong absorption of such $\gamma$ rays passing within the region of the inner few tens of stellar radii around the star. Some photons might however undergo a few generations of an electromagnetic cascade and thus still be able to escape this region.

In Fig. 2 2 we study the dependence of the observed emission on various parameters of the relativistic electrons. For simplicity we normalise the escaping $\gamma$-ray emission to 1 erg of energy injected in the blob (measured in its frame). The emission is integrated over the whole range of the observation angles. In the top left panel, we investigate how the $\gamma$-ray spectrum depends on the impact parameter of the primary electrons. For electrons passing close to the star, the $\gamma$ ray absorption in the stellar radiation makes a clear imprint on the spectrum. Interestingly, even while the optical depths for $\mathrm{TeV}$ photons passing next to the star reach values $\gtrsim 10$, there is still a measurable flux of $\mathrm{TeV}$ photons emitted for electrons passing that close to the star surface. It is a combined effect of the cascading process and the fact that the impact parameters of electrons are usually smaller than those of secondary $\gamma$-rays. To explain the second effect, let us consider a $\gamma$ ray produced by an electron in a blob approaching a star $(d \approx 0)$ at the distance $L$. Due to the anisotropy of the electron direction the $\gamma$ ray is expected to be produced at an angle $\theta_{\gamma} \sim 1 / \gamma_{b}$ with respect to the blob movement direction. Thus, the impact parameter of the $\gamma$ ray, $\sim L \theta_{\gamma}$, might be much larger than the impact parameter of its parent electron, provided that the $\gamma$ ray was emitted far from the star. Moreover, if the optical depth is of the order of 10 , the $\gamma$-ray might still escape the production region with similar energy, undergoing a few generations of an electromagnetic cascade if the IC process occurs in KN regime (compare the thin and thick red curve in Fig. 3, on the left). The cascading effects becomes negligible for the impact factor of an electron clearly exceeding the value of $\sim 10 R_{\star}$. In Fig. 目 (second figure), we investigate the dependence of the $\gamma$-ray spectra on the Lorentz factor of the blob. As the electron energy is normalised to a value calculated in the blob's frame of reference, for large values of $\gamma_{b}$ the emission is magnified by $\sim \gamma_{b}$ and extends to 
higher energies. As expected, the spectral shape also depends strongly on the spectral index, $\alpha$, of the power law spectrum of electrons (see Fig. 2. third figure). For very flat electron spectra, the visibility of the absorption dip is influenced by the maximum energies of electrons (see Fig. 3 on the right).

\section{Interpretation of PKS 1222+21}

PKS $1222+21(4 \mathrm{C}+21.35)$ is a flat spectrum radio quasar (FSRQ) at the distance corresponding to the redshift $z=0.432$ [12]. The jet moves at a small angle to the observer's line of sight with the apparent superluminal motion $\beta_{\text {app }}>10$ [13, 14, 15]. PKS 1222+21 has been detected by FermiLAT in the GeV energy range [16, 17] and also by AGILE [18]. First two years of the Fermi-LAT observations of PKS 1222+21 has been studied in detail in [19]. Two large outbursts have been observed from this source in April and June 2010. The $\gamma$-ray spectrum is well described by the power law with the differential spectral index close to -2 below 1-3 GeV with a clear steepening above this energy (spectral index in the range $\sim 2.4-2.8$, [19]). During the second Fermi-LAT detected flare the source was also discovered by the MAGIC Collaboration in the energy range 70$400 \mathrm{GeV}$ [20]. The observed MAGIC spectrum is well fitted by the power law with a spectral index $3.75 \pm 0.27_{\text {stat }} \pm 0.2_{\text {syst }}$. The sub-TeV emission has shown a rapid variability with the doubling time of the order of $\sim 10 \mathrm{~min}$ [20]. A rather weak synchrotron emission of PKS $1222+21$ is partially obscured by a stronger emission from the accretion disk and the dusty torus (e.g. [21]). The isotropic power emitted in $\gamma$-rays is $L_{\gamma} \cong 7 \times 10^{47} \mathrm{erg} \mathrm{s}^{-1}$ ([19]). The mass of the black hole within PKS 1222+21 is expected to be in the range $6-8 \times 10^{8} \mathrm{M}_{\odot}[22,21]$. The above described emission features are difficult to understand in terms of any proposed models for the $\gamma$-ray production (see e.g. discussion in [19, 20, 21]).

Here we propose that the $\gamma$-ray flare can be interpreted in terms of the model discussed above, i.e. as a result of a collision of the relativistic blob in the jet with a luminous star. In fact the low energy spectrum, in the X-rays and the part below UV, does not change significantly between low and high $\gamma$-ray states (see e.g. Fig. 9 in [21]). But the GeV-TeV $\gamma$-ray emission changes by over an order of magnitude. Such spectral behaviour resembles emission observed during the so called orphan $\gamma$-rays flares reported earlier from e.g. BL Lac type active galaxy 1ES 1959+650 [28].

As an example, we interpret the $\gamma$-ray emission from the flare observed by the Fermi-LAT and MAGIC as a result of an encounter of a luminous $O$ type star by a relativistic blob in the jet. The time scale of the flare observed by the MAGIC telescopes puts a strong constraint on the longitudinal shape and extend of the blob. We assume in the calculations a Gaussian distribution of the electrons with a standard deviation (measured in the reference frame of the star) of $H_{b}=$ $13 R_{\star}$. Such a shape results in a $\gamma$-ray light curve consistent with the one observed by the MAGIC telescopes (see Fig. 3 on the left).

We calculate the corresponding $\gamma$-ray spectrum constructed from the photons arriving at the observer within the observation time of MAGIC and plot it in Fig. 3 on the right. Although it is possible to explain the very fast variability of the emission even with a moderate Lorentz factor of the blob, strong constraints are put by the level of the observed flux. If the emission region has a radius of $R_{\mathrm{b}}=3 \times 10^{14} \mathrm{~cm}$, and is moving with a Lorentz factor of $\gamma_{\mathrm{b}}=100$, we require an energy density of $\rho_{E}=20 \mathrm{erg} \mathrm{cm}^{-3}$ (measured in the blob's frame of reference) to reproduce 

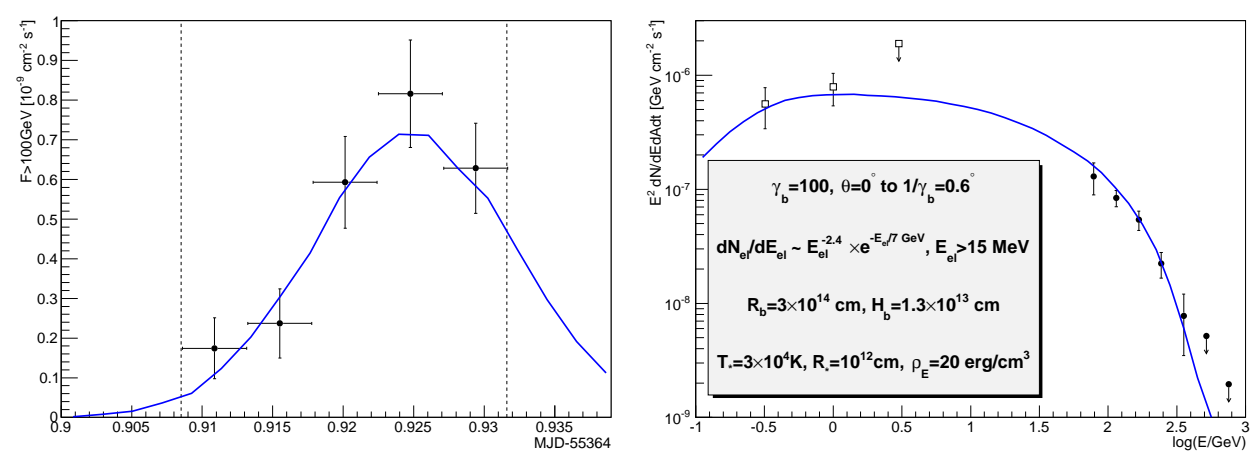

Figure 3: Interpretation of the $\gamma$-ray emission observed during the flare from the FSRQ PKS 1222+21 in June 2010 by Fermi-LAT (empty squares) and MAGIC (full circles), [2]]. The absorption in the Extragalactic Background Light is taken into account according to [24] model. The left figure shows the light curve above $100 \mathrm{GeV}$. The dashed vertical lines are the time range from which the spectral energy distribution (the right figure) is computed. The parameters of the blob, star and electron distribution are given in Sect. 3. (reproduced from [9])

the flux observed by MAGIC and Fermi-LAT (Fig. 3 on the right). Note that such large values of the Lorentz factor of the emission region in the jet have been already postulated in terms of other models in order to explain extremely short flares observed in this source ([21]) or in the other sources, e.g. PKS 2155-304 [25]. Such large Lorentz factors of the blob find also some observational support from the observations of the superluminal motion in PKS 1510-089 which represents similar type of blazar [26]. We can estimate the power of the blob in the observer's reference frame on, $L_{\mathrm{blob}}=\pi R_{\mathrm{b}}^{2} c \rho_{E} \gamma_{\mathrm{b}}^{2} \approx 1.7 \times 10^{45} \mathrm{erg} \mathrm{s}^{-1}$. On the other hand, the Eddington luminosity of the black hole in PKS $1222+21$, with the mass $6-8 \times 10^{8} \mathrm{M}_{\odot}$, is $L_{\mathrm{Edd}}=1.3 \times$ $10^{47} M_{9} \approx 8-10 \times 10^{46} \mathrm{erg} \mathrm{s}^{-1}$. Therefore, the blob has to contain about $\sim 2 \%$ of the Eddington power. This is quite demanding but seems not to be excluded, especially if $R_{\mathrm{b}} \approx R_{\perp}$. Lower values of $\gamma_{b}$ require a much higher energy density in the blob (e.g. $\rho_{E}=340 \mathrm{erg} \mathrm{cm}^{-3}$ for $\gamma_{b}=50$ and $R_{\mathrm{b}}=3 \times 10^{14} \mathrm{~cm}$ ). The strong dependence on the $\gamma_{\mathrm{b}}$ is a combined result of the transformation of the energy density to the reference frame of the observer and the beaming of the emission in a narrower cone. Note that a larger radius of the blob will lower the energy density constrain, e.g. for $R_{\mathrm{b}}=10^{15} \mathrm{~cm}$ we obtain $\rho_{E}=4.9 \mathrm{erg} \mathrm{cm}^{-3}$ for $\gamma_{\mathrm{b}}=100$ and $\rho_{E}=80 \mathrm{erg} \mathrm{cm}^{-3}$ for $\gamma_{\mathrm{b}}=50$, at the assumption that there is no competing energy loss process of the electrons at such a large distance from the star.

\section{Conclusion}

We propose that blobs of relativistic plasma, moving in jets of AGNs, can encounter from time to time luminous stars which form a quasi-spherical halo around the central super-massive black hole. The transit time of a star through a conical jet can be estimated as $T_{\text {transit }}=R_{\perp} / \nu_{\star} \approx$ $0.45 \gamma_{30}^{-1} l_{-1}^{3 / 2} / M_{9}^{1 / 2} \mathrm{yr}$, where the velocity of the star is $v_{\star}=\sqrt{G M_{\mathrm{BH}} / l} \approx 7 \times 10^{8}\left(M_{9} / l_{-1}\right)^{1 / 2} \mathrm{~cm}$ $\mathrm{s}^{-1}, M_{\mathrm{BH}}=10^{9} M_{9} \mathrm{M}_{\odot}$ is the black hole mass in units of the Solar mass, and $G$ is the gravitational constant. The star passing the inner jet at parsec distance from the SMBH can be responsible for a sequence of outbursts as different blobs reach its position. Such high activity period can last from 
months to years. In fact, a single blob may be also responsible for the observed sequence of flares (e.g. as observed in Mrk 421 [27] or PKS 2155-304 [25]), if it meets on its path a few stars within a jet.

Luminous stars are also characterised by stellar winds which pressure can balance the pressure of the blob plasma. As a result a shock structure appears around the star. The shock radius around the star in a conical jet can be determined from (see e.g. [1]), $R_{\mathrm{sh}}^{\star} \approx 1.9 \times 10^{12}\left(M_{-5} v_{3}\right)^{1 / 2} \gamma_{30}^{-1} l_{-1} / L_{46}^{1 / 2}$ cm, where $\dot{M}=10^{-5} M_{-5} M_{\odot}$ and $v=10^{3} v_{3} \mathrm{~km} \mathrm{~s}^{-1}$ are the mass loss rate and the velocity of the stellar wind, and $L_{\mathrm{b}}=10^{46} L_{46} \mathrm{erg} \mathrm{s}^{-1}$ is the power of the blob in the observer's frame. Since the surface area of this shock, $\sim \pi R_{\mathrm{sh}}^{2}$, is typically much smaller than the surface area of the considered blob, the presence of the shock should not essentially influence presented above calculations of the $\gamma$-ray emission from the blob.

Our model predicts the appearance of flares which emission is limited mostly to the $\gamma$-ray energy range. Therefore, it can provide the mechanism for the appearance of the so-called orphan $\gamma$-ray flares observed occasionally from relatively nearby AGNs of the BL Lac type (e.g. 1ES 1959+650 [28] or Mrk 421 [29]) but also from the FSRQs (e.g. PKS 1222+21 [20]). In order to show applicability of this scenario we model the case of the emission from the very strong $\gamma$ ray flare observed from the distant FSRQ PKS 1222+21 in 2010. Our model is able to reproduce the observed light curve and spectral energy distribution measured by the Fermi-LAT and MAGIC, although the energy requirements are quite demanding. Note that in the case of blazars with orphan flares which are about an order of magnitude closer to the observer (i.e. 1ES 1959+650 and Mrk 421), this energy requirements should be about two orders of magnitude lower or the Lorentz factors of the blob might be closer to values typically reported from radio observations.

\section{Acknowledgements}

This work is supported by the grant through the Polish Narodowe Centrum Nauki No. 2015/19/N/ST9/01727

\section{References}

[1] W. Bednarek, R.J. Protheore, Gamma-rays from interactions of stars with active galactic nucleus jets, MNRAS 287 (1997) L9

[2] M.V. Barkov, F.A. Aharonian, V. Bosch-Ramon, V., Gamma-ray Flares from Red Giant/Jet Interactions in Active Galactic Nuclei, ApJ 724 (2010) 1517

[3] V. Bosch-Ramon, M. Perucho, M., M.V. Barkov, Clouds and red giants interacting with the base of AGN jets, A\&A $\mathbf{5 3 9}$ (2012) 69

[4] A.T. Araudo, V. Bosch-Ramon, G.E. Romero, Gamma-ray emission from massive stars interacting with active galactic nuclei jets, MNRAS 436 (2013) 3626

[5] S. Wykes et al., Filaments in the southern giant lobe of Centaurus A: constraints on nature and origin from modelling and GMRT observations, MNRAS 442 (2014) 2867

[6] W. Bednarek, P. Banasiński, Non-thermal Radiation from Collisions of Compact Objects with Intermediate-scale Jets in Active Galaxies, ApJ 807 (2015) 168

[7] V. Bosch-Ramon, Non-thermal emission from standing relativistic shocks: an application to red giant winds interacting with AGN jets, A\&A $\mathbf{5 7 5}$ (2015) A109

[8] V.M. de la Cita et al., Coupling hydrodynamics and radiation calculations for star-jet interactions in active galactic nuclei, A\&A 591 (2016) 15 
[9] P. Banasiński, W. Bednarek, J. Sitarek, Orphan gamma-ray flares from relativistic blobs encountering luminous stars, MNRAS 463 (2016) L26

[10] K. Asada, M. Nakamura, The Structure of the M87 Jet: A Transition from Parabolic to Conical Streamlines, ApJ 745 (2012) L28

[11] J. Sitarek, W. Bednarek, Time-dependent gamma-ray production in the anisotropic inverse Compton electron-positron pair cascade initiated by electrons in active galaxies, MNRAS 409 (2010) 662

[12] D.E. Osterbrock, R.W. Pogge, Optical spectra of narrow emission line Palomar-Green galaxies, ApJ 323 (1987) 108

[13] J.R.A. Hooimeyer et al., Two new candidate superluminal quasars - 4C 28.45 and 4C 21.35, A\&A 261 (1992) 5

[14] S.G. Jorstad et al., Multiepoch Very Long Baseline Array Observations of EGRET-detected Quasars and BL Lacertae Objects: Superluminal Motion of Gamma-Ray Bright Blazars, ApJS 134 (2001) 181

[15] D.C. Homan et al., Parsec-Scale Blazar Monitoring: Proper Motions, ApJ 549 (2001) 840

[16] F. Longo, M. Giroletti, G. Iafrate, G., Fermi LAT detection of increasing gamma-ray activity of blazar PKS 1222+216, ATel. 2021 (2009)

[17] S. Ciprini ATel. 2349 (2009)

[18] G. Iafrate, F. Longo, F. D’Ammando, Fermi LAT detection of a very intense GeV flare from 4C +21.35(PKS 1222+21), ATel. 2687 (2010)

[19] Y.T. Tanaka et al., Fermi Large Area Telescope Detection of Bright Gamma-Ray Outbursts from the Peculiar Quasar 4C +21.35, ApJ 733 (2011) 19

[20] J. Aleksić et al., MAGIC Discovery of Very High Energy Emission from the FSRQ PKS 1222+21, ApJ 730 (2011) L8

[21] M. Ackermann et al., Multifrequency Studies of the Peculiar Quasar 4C +21.35 during the 2010 Flaring Activity, ApJ 786 (2014) 157

[22] E.P. Farina et al., The optical spectrum of PKS 1222+216 and its black hole mass, MNRAS 424 (2012) 393

[23] H. Krawczynski et al., Multiwavelength Observations of Strong Flares from the TeV Blazar 1ES 1959+650, ApJ 601 (2004) 151

[24] A. Domínguez et al., Extragalactic background light inferred from AEGIS galaxy-SED-type fractions, MNRAS 410 (2011) 2556

[25] F. Aharonian et al., An Exceptional Very High Energy Gamma-Ray Flare of PKS 2155-304, ApJ 664 (2007) L71

[26] S.G. Jorstad et al., Polarimetric Observations of 15 Active Galactic Nuclei at High Frequencies: Jet Kinematics from Bimonthly Monitoring with the Very Long Baseline Array, AJ 130 (2005) 1418

[27] J.H. Buckley et al., Gamma-Ray Variability of the BL Lacertae Object Markarian 421, ApJ 472 (1996) L9

[28] H. Krawczynski et al., Multiwavelength Observations of Strong Flares from the TeV Blazar 1ES 1959+650, ApJ 601 (2004) 151

[29] N. Fraija et al. 2015, In proceedings of "Swift: 10 Years of Discovery" congress (Rome, 2-4 December 2014), PoS(SWIFT 10)152 